\title{
Positive Changes in Life Outcomes over the Pandemic in Chinese Adolescents: The Role of Resilience and Relation to Mental Health
}

Jian-Bin Li

The Education University of Hong Kong

Kai Dou ( $\square$ psydk@gzhu.edu.cn )

Guangzhou University https://orcid.org/0000-0002-2449-7969

Zi-Hao Liu

Guangzhou University

Research article

Keywords: positivity, benefits, life outcomes, adolescent development, COVID-19, pandemic, latent profile analysis

Posted Date: November 8th, 2021

DOI: https://doi.org/10.21203/rs.3.rs-1038286/v1

License: (1) This work is licensed under a Creative Commons Attribution 4.0 International License. Read Full License 


\section{Abstract \\ Background}

Although the existing literature has well documented the negative effects of COVID-19 on multiple life outcomes in adolescents, some research has also revealed that some life outcomes have become better during COVID-19. Scant research has specifically examined to what extent and in what aspects COVID-19 is beneficial to adolescent development so far. With person-centered approach, this research addressed this gap by: (1) exploring different profiles of positive changes in life outcomes in Chinese adolescents since the outbreak of COVID-19; (2) examining the role of resilience in relation to different profiles; (3) comparing mental health across adolescents categorized into different profiles.

\section{Method}

Participants were 2,567 Chinese adolescents aged 12 to 24 . They rated how much their lives of different domains had experienced positive changes since the outbreak of the pandemic. They also answered the questionnaires that measured their resilience and mental health.

\section{Results}

Results of latent profile analysis revealed three different profiles: limited positive changes (33.3\%), partial positive changes (49.5\%), and overall strong positive changes (17.2\%). Moreover, adolescents with a higher level of resilience were more likely to be categorized into the partial positive changes profile compared to the limited positive changes profile and into the overall strong positive changes profile compared to the other two profiles, after controlling for the covariates. Finally, adolescents in the overall strong positive changes profile had better mental health than their counterparts in the other two profiles.

\section{Conclusion}

COVID-19 might be helpful to adolescent development to some extent, especially for those with higher resilience.

\section{Background}

The outbreak of coronavirus disease (COVID-19) has affected most people globally (1). Governments are implementing strict measures against the spread of the coronavirus, such as lockdowns, study/work from home, physical distancing, limitation of social interactions, and quarantine. Despite its effectiveness to stop the coronavirus, the measures also have side effects such as increasing people's isolation and stress (2). Adolescents, the population aged 10 to 24 (3) [i], are considered particularly vulnerable to the social effects of the measures against COVID-19 (4). Adolescence is a developmental period marked by rapid physical and social-emotional changes, onset of many mental disorders, and increased time investment with peers $(5,6)$, but the measures have disrupted adolescents' life structure and may result in multiple negative life outcomes. Research has well documented the negative effect of COVID-19 on adolescents' psychosocial outcomes, such as poorer mental health, decreased well-being, reduced physical activity, more alcohol use, and increased screen time (7-10).

Nevertheless, some studies also unexpectedly found that COVID-19 induced positive changes in some life outcomes in adolescents, such as increased psychosomatic health and more time to sleep $(8,9)$. These findings lead to an intriguing, yet rarely explored, question: can COVID-19 be beneficial to adolescents' life outcomes? A recent review has discussed this question, postulating that successfully navigating the challenges of COVID-19 would strengthen adolescents' sense of community, family cohesion, and personal growth and mental health (4). The study further considered that young people's ability to cope with the pandemic is determined by demographic, social (e.g., social support), and individual (e.g., ability to make meaning from the crisis) factors.

Only a few studies have examined positive changes in life outcomes in adolescents during COVID-19. One research examined this topic during the initial lockdown in Scottish adults (11). It found that the participants reported positive changes in various life outcomes, with higher levels in some aspects (e.g., more appreciative of things usually taken for granted) than in others (e.g., spent less time on screen or devices outside of work hours). The results also found that females, younger people, and individuals with better health reported higher 
levels of positive changes. This study, however, did not focus on adolescents and it did not examine the individual antecedents or mental health outcomes. Moreover, the effects on adolescents' positive changes in life outcomes may differ across countries, as the measures adopted by different countries differ in intensity, effectiveness, and duration. However, it is unknown whether adolescents from other countries (e.g., China) would experience similar patterns of positive changes. As people's psychological functioning may manifest in different profiles when the pandemic has evolved for a period of time, person-centered approach with multiple indicators is considered an appropriate method to capture this complex patterns (12).

Therefore, the aim of this study was threefold. First, we explored different profiles of positive changes in life outcomes in Chinese adolescents over the past two years in a national sample $(N=2,567)$. This topic is exploratory in nature, but we expected that distinct profiles would emerge. Second, we examined the extent to which resilience, defined as one's ability to bounce back from external stressor (13), would be related to the membership of profiles. Given that resilience is an important asset that helps young people deal with crisis such as COVID-19 (14), we hypothesized that adolescents with high levels of resilience would be more likely to be in a profile indicating more positive changes. Third, we examined mental health as a function of profiles. Based on prior viewpoints (4), we hypothesized that adolescents in a more positive profile would have better mental health than those in a less positive profile.

[i] We pre-registered that this study would focus on adolescents aged 12 to 25 . But in this study we decided to focus on adolescents aged 12 to 24 to follow the definition of adolescents by Sawyer et al. (2018).

\section{Method}

\section{Participants and Procedure}

Participants of this study were from a larger survey that investigated Chinese public's intention/motivation of getting COVID-19 vaccine and its correlates. Student helpers $(N=168)$ from various regions in China volunteered to spread an online survey link on their social networks via different social media platforms (e.g., WeChat). Within the survey period (from 23-July to 12-August, 2021), 3,273 participants provided consent and completed the survey. As a quality control, we excluded a number of participants because: (1) their age was out of our expected range ( $<12$ or $>90$ years old; $N=11)$; (2) they were living oversea $(N=7)$; (3) they had duplicate IP address $(N=195)$, or (4) they answered the survey too fast according to Leiner's (2019) relative speed index $(>2 ; N=75)[i](15)$. The remaining 2,985 participants constituted the final sample of the larger survey ( $M_{a g e}=22.07$ years; 1,019 males).

Adolescents aged 12 to 24 from the said larger survey consisted of the sample of this study. The current sample size was 2,567 adolescents from 32 provinces/regions in China (850 males; $M_{a g e}=19.87$ years, $S D=2.02$ ). Among them, 157 and 28 participants reported that they had history of physical or psychiatric illness, respectively; and 44 participants reported that they were directly related to COVID-19 (e.g., confirmed/suspicious cases and/or relatives/friends of the confirmed cases, etc.).

This study was approved by the ethical committee of xx University. The survey was conducted online. Participants (or their guardians) provided consent by checking the box in the front page of the survey that fully explained the study. They reserved the right to withdraw from the survey at any point. Participation was voluntary and no incentive was provided. We did not collect identifiable personal particulars and confidentiality was stressed.

\section{Measures}

\section{Positive Changes in Life Outcomes}

We adapted the positive events subscale of the Epidemic-Pandemic Impacts Inventory to measure used in previous study to measure Chinese adolescents' positive changes in life outcomes(11). The previous study used 21 items measuring whether participants had experienced positive changes during the initial lockdown across a number of life outcomes (e.g., relationships, physical activity, sleep, work) with binary response options (11). In this study, we modified this subscale in several aspects to suit the current participants better. First, we grouped some items that had similar meaning. For instance, in the original subscale, the items "more quality time with partner or spouse" and "more quality with children" were combined and modified as "more quality time with family members". Second, social media information was filled with positive energy (e.g., sharing of gratitude, appreciation, social responsibility)(16) and patriotism (17) in China over the last two years when the government was combating COVID-19. In this regard, we added a few items that reflected this situation, such as "become more gratitude" and "become more patriotic". Finally, we changed the binary response options to a five-point scale (from $1=$ strongly disagree to $5=$ strongly agree). A higher score indicates higher levels of positive changes in life outcomes. The final scale has 19 items. Participants were asked to rate how much they had experienced the listed positive changes in various life 
outcomes since the outbreak of COVID-19. The full set of items are illustrated in Table 2. The Cronbach's a of this scale was 0.95 in this study.

\section{Resilience}

We used the Brief Resilience Scale (13) to measure participants' ability to bounce back. In this study, the Chinese version of BRS was used (12). This scale has 6 items rated on a five-point scale (from $1=$ strongly disagree to $5=$ strongly agree). A higher mean score indicates better ability to bounce back from stressors. Sample items are "It does not take me long to recover from a stressful event" and "I usually come through difficult time with little trouble". The Cronbach's a of this scale was 0.69 in this study.

\section{Mental Health}

We used GHQ-12 (18) to measure participants' mental health. The Chinese version of this scale was used (19). This measure consists of 12 items which are either stated in negative or in positive wordings. Participants were asked to rate on these items according to their situation over the past month compared to their usual situation. All items are rated on a four-point scale (from 1=better than usual to 4=much worse than usual). A higher mean score indicates more mental health problems (i.e., less mental health). Sample items are "feel unhappy and depressed" and "Iose confidence in self". The Cronbach's a of this scale was 0.90 in this study.

\section{Covariates}

We measured several demographic variables as covariates, as they had been found to be associated with various life outcomes in Chinese public at the beginning period of COVID-19 (20). These covariates include biological sex ( $1=$ male, $2=f e m a l e)$, age, their relationship with COVID-19 (1=directly related, such as confirmed/suspicious case and/or relatives/friends of the confirmed cases, etc., $2=$ not related), history of physical and psychiatric illness $(1=y e s, 2=n o)$, and their current physical health condition (from $1=v e r y$ poor to $5=$ very good).

\section{Data Analyses}

We analyzed the data with SPSS 26.0 and Mplus 7.31 (21). The variables examined in this study had never been used in other studies. The research question, hypotheses, and the data analytic plan were pre-registered at aspredicted.org (protocol number: \#73896). First, we conducted preliminary analyses, including means and standard deviation. Second, we carried out latent profile analysis (LPA) to examine the first research question. All the 19 items listed in Table 2 were used as the indicators of analysis. We first started with one-profile, and then increased the number of profiles systematically until we identified the best fitting model according to a number of indices, including Akaike Information Criteria (AIC)(22), Bayesian Information Criterion (BIC)(23), adjusted BIC (aBIC), Lo-Mendell-Rubin Adjusted Likelihood Ratio Test (LMRT)(24), and Bootstrapped Likelihood-Ration Test (BLRT)(25). Smaller values of AIC, BIC, and aBIC indicated better model fit. The $p$-value values associated with LMRT and BLRT indicate whether the $k$-profile model $(p<.05)$ or the $k$ - 1 profile model $(p>05)$ has a better fit. Besides, the value of entropy no less than 0.6 indicates good profile separation (26). In addition, we also considered theoretical meaningfulness of the profile (27) and the proportion of participants represented in the profiles (28). As a rule of thumb, no profile should have a group comprised of less than $5 \%$ of the participants (29). Multivariate analysis of variance (MANOVA) was conducted to examine whether the mean level of each indicator significantly differed across profiles. $P$-values and effect sizes $\left(\eta^{2}{ }_{p}\right)$ were used to judge the significance. Third, we performed a logistic regression model using a three-step procedure in Mplus (i.e., R3STEP auxiliary command)(26) to examine the second research question after identifying the best-fitting model. Logits from the model output were transformed into odd ratios for explanation purposes. Demographic variables measured above were included in the model as covariates. Fourth, analysis of variance (ANOVA) was performed to examine the third research question, with the average score of GHQ as the dependent variable and the profiles as the independent variables. Both $p$-values and effect size $\left(\eta^{2}{ }_{p}\right)$ were used to determine the significance. Based on ANOVA, we further conducted ANCOVA controlling for covariates. Finally, as a robust check, we replicated the analyses for research questions 2 and 3 with winsorized scores of resilience and GHQ[ii], respectively.

[i] Leiner's (2019) relative speed index was calculated in the following steps. First, although the questionnaires administered to participants who had and who had not got vaccinated were largely the same, several questionnaires were different, thus resulting in different completion time between the two groups of participants. Hence, we split the two groups and gauged the relative speed index of each participant for each group. Second, we calculated the median completion time for each group. Third, we used each participant's completion time to divide the median completion time of the group he/she was in, and a relative speed index could be obtained. For instance, if the median completion time of the non-vaccinated group was 600 seconds, a participant in this particular group had a 
completion time of 200 seconds. Then, this participant's relative speed index was $600 / 200=3$ and he/she should be excluded because his/her index was larger than the cut-off point (i.e., 2).

[ii] Winsoring approach (Tukey, 1962) was used to handle the outliers of the resilience and GHQ by replacing the outliers with the nearest number within the -3 to +3 SD range.

\section{Results}

\section{Preliminary Analysis}

Descriptive analysis found that participants overall reported above-medium levels of positive changes in life outcomes ( $M=3.74$, $S D=0.61)$. Besides, participants reported medium level of ability to bounce back $(M=3.17, S D=0.55)$ and low levels of mental health problems $(M=1.95, S D=0.49)$.

\section{Profiles of Positive Changes in Life Outcomes}

As shown in Table 1, latent profile analysis found that the 3-profile solution described the optimal number of profiles for life changes. According to the fit indices, the 3-profile solution demonstrated a better fit than the 2-profile solution, as indicated by significant LMRT ( $p$ $<.001)$ and BLRT $(p<.001)$. By contrast, the 4-profile solution was not better than the 3-profile solution, as indicated by insignificant LMRT ( $p=.533$ ). Besides, the values of AIC, BIC, and aBIC decreased as the number of profiles increased, and such decrease appeared more apparent between 1-profile and 2-profile solutions and between 2-profile and 3-profile solutions than the one between 3-profile and 4-profile solutions. Moreover, no group contained less than $5 \%$ of the participants for the 3-profile solution, but one group of the 4-profile solution contained less than $5 \%$ of the participants. Taken together, we selected the 3 -profile solution as the final solution. This solution showed high entropy (i.e., 0.93). The average posterior profile membership probability was high, with $96.7 \%, 97.1 \%$, and $97.2 \%$ for the first, second, and the third profile, respectively.

Table 2 presents the raw score of each indicator of each profile and the results of MANOVA. We labelled the first profile as "limited positive changes". This profile consisted of $33.3 \%$ of participants. Participants in this profile showed positive changes in only a few aspects of life outcomes, mainly concerning personal health (e.g., item $3 \& 14$ ) and social responsibility (e.g., items $13 \& 15)$ and such changes were moderate, as their scores were not far from the mid-point (i.e., 3.00). We labelled the second profile as "overall strong positive changes". This profile contained about $17.2 \%$ of the participants. Participants in this profile reported positive changes in all the listed aspects of life outcomes at high level (i.e., >4.00). We labelled the third profile as "partial positive changes". This profile contained about half of the participants (49.5\%). Participants in this profile showed positive changes in several aspects related to self-care (e.g., items 4, 5, \& 14), positive virtues (e.g., items 7 \& 12), social responsibility (e.g., items 11, 13 \& 15), and emotional and psychological wellbeing (e.g., items 17 to 19). The magnitudes of changes in these aspects were higher than the mid-point but did not reach high levels $(>4.00)$.

We conducted a MANOVA to examine whether the indicator scores were different across the three profiles. The results indicated a significant multivariate test, Wilk's $\lambda=0.17, F(38,5092)=188.61, p<.001, \eta_{p}^{2}=.59$. Results of between-subject effects revealed significant main effect for each indicator. Post-hoc multiple comparison with Bonferroni correction found that the over strong positive changes profile had a significantly higher level on each indicator than the other two profiles and that the partial positive changes profile in turn had a significantly higher level of each indicator than the limited positive changes profile.

\section{The Association between Resilience and Different Profiles}

The results of logistic regression are summarized in Table 3. As shown, compared to the limited positive changes profile, participants with 1 unit increase in resilience were 2.01 and 1.43 times more likely to be the members of the overall strong positive changes and the partial positive changes profile, respectively, net the effects of covariates. Moreover, compared to the partial positive changes profile, participants with 1 unit increase in resilience were 1.40 times more likely to be the members of the overall strong positive changes profile, even after controlling for covariates. In addition, replication with winsorized resilience score as the predictor yielded very similar findings.

\section{The Differences in Mental Health among Different Profiles}

The results of ANOVA analysis found a significant main effect across the limited positive changes $(M=2.09, S D=0.45)$, partial positive changes $(M=1.91, S D=0.44)$ and overall strong positive changes $(M=1.79, S D=0.62)$ profiles, $F(2,2564)=65.77, p<.001, \eta^{2} p^{2}=.05$. Post-hoc multiple comparison with Bonferroni correction found that the adolescents in the overall strong positive changes profile had a 
significantly lower level of mental health problems than those in the other two profiles and that adolescents in the partial positive changes profile had a significantly lower level of mental health problems than those in the limited positive changes profile. We further conducted ANCOVA analysis controlling for covariates, the effect of profile on mental health problems remained significant, $F(2$, 2558) $=32.10, p<.001, \eta^{2}=.02$. In addition, replications with winsorized GHQ-12 score as outcome yielded very similar findings for both ANOVA and ANCOVA analyses.

\section{Discussion}

This research generated three findings. First, three qualitatively distinct profiles of positive changes in life outcomes emerged in Chinese adolescents. Second, adolescents with a higher level of resilience were more likely to be in a more positive profile than those with a lower level of resilience. Third, more positive changes in life outcomes were related to better mental health. Altogether, these results advance our understanding of an underexplored research topic: to what extent and in what aspects COVID-19 is beneficial to adolescent development.

Previous studies found that while COVID-19 deteriorated some life outcomes (e.g., screen time, mental health, and well-being) it increased other outcomes (e.g., psychosomatic health and sleep time) $(8,9)$. The emergence of three different profiles of this study reconcile the said inconsistent findings. A recent review has pointed out that people's adjustment outcomes may vary across domains and over time during COVID-19, with some people showing better outcomes in some aspects at a certain period and other people in other aspects at a different period (12). Our findings are consistent with this viewpoint. As illustrated in Table 2, some participants showed positive changes in more aspects and reported higher levels of positive changes than others. This finding is also consistent with prior research that found that Scottish public generally reported positive changes in multiple life domains but that the intensity of changes varied across domains (11).

Of note, participants across profiles showed relatively higher positive changes in life outcomes related to personal health and survival (e.g., pay more attention to physical health) and to social and ideological issues (e.g., become more patriotic). According to terror management theory (30), the former may reflect that adolescents manage the anxiety induced by the pandemic through actively engaging in thoughts and behaviors that prolong their survival. The latter may result from the (positive) information spread among the Chinese public during COVID-19 (16). Moreover, participants across profiles showed relatively lower levels of positive changes in issues related to routines (e.g., spent less time on screen and sleep quality). This may be because school closure has been enforced and online learning via digital devices has been adopted from time to time in different regions over the past two years. Regarding screen time, research found increased in frequency and duration of recreational internet use in Chinese children and adolescents, especially among boys (31), probably because of more access to digital device during the pandemic. In sum, these findings implied that the preventive measures appeared to affect Chinese adolescents' routine quite extensively.

As predicted, adolescents with a higher level of resilience were more likely to be in the profile indicating more positive changes. According to the risk-resilience model, individuals with more personal or social assets would better cope with stressors to maintain adjustment (32). In support of this idea and prior findings $(13,14)$, the current findings suggest that resilience is an essential personal asset that helps adolescents navigate the stressors during COVID-19 and achieve more positive changes. One possible explanation may be because resilience supports adolescents' reflective functioning, which is an essential factor that facilitates effective meaning-making from stressful events and personal growth (33-35). A clinical implication of this finding is that enhancing adolescents' ability to bounce back might be a promising way to help them achieve personal growth during the pandemic.

Adolescents perceiving overall strong positive changes in multiple life outcomes over the past two years showed better mental health over the past month, which confirmed our hypotheses. This finding is not surprising, as participants who are resilient, successfully recover from, and effectively make meaning of the pandemic are more likely to have better adjustment, such as mental health (12).

This research has several limitations. First, the life outcomes examined in this research are not exhausted, although these outcomes are appropriate to Chinese adolescents and most of them have been examined in prior research (11). Second, this sample was not nationally representative, as girls were over represented according to Chinese census (36) and the sample size of different regions differed considerably. Third, utilizing longitudinal design to examine adjustment during COVID-19 is promising as it allows direct comparison across periods (12), but the design of this research is cross-sectional in nature. Nevertheless, we reckon that the current findings are still valuable in that they reflect adolescents' overall perception of the positive changes in life outcomes over the past two years. These results are meaningful, as scant research has specifically examined the positive psychosocial effect of COVID-19 on adolescent development so far. Future research may continue examining this topic with more sophisticated measurement, more representative 
samples, and longitudinal design. In addition, cross-cultural comparison would be also promising, as how adolescents perceive their lives are affected by COVID-19 could be determined by the situations of the pandemic and the effectiveness as well as the impacts of the measures used to stop the virus in different nations.

Does COVID-19 only induce negativity? Our findings reveal that Chinese adolescents have generally perceived positive changes in different domains of life outcomes over the past two years, although some of them perceive positive change in more life domains and at higher levels than others. Resilience is a vital factor associated with adolescents' perceived positive changes. Finally, positive changes in more domains and at higher levels are related to better mental health. Altogether, these findings imply that COVID-19, as an external stressor, might be helpful to adolescent development to some extent, especially for those who are more psychologically resilient. Given the scarce of this topic, we encourage continuous research on this topic.

\section{Conclusion}

Despite the negative psychosocial effects induced by COVID-19 on adolescent development, it is also necessary to consider the positive side it brings. Most adolescents report positive changes in life outcomes of various domains at different levels. Helping adolescents see the positive sides from the crisis, such as through improving their ability to bounce back, would lay the foundation for recent mental health.

\section{Declarations}

\section{Acknowledgements}

None.

\section{Authors' contribution}

LJB and DK: Study conceptualization and design, data analysis, manuscript drafting and revision. LZH: Data collection and data entry. LJB and DK: Data analysis, reviewed, and edited the manuscript. All authors have agreed to authorship and order of authorship for this manuscript.

\section{Funding}

This work was supported a grant by the Guangzhou Education Scientific Research Project (Grant No.202113700).

\section{Availability of data and materials}

The measures are attached as supplementary files and the dataset is available from the corresponding authors upon request.

\section{Ethics approval and consent to participate}

The study received ethical approval from the Ethics Review Committee of Education School, Guangzhou University [GZHU2020001] and all participants provided consent to participate online.

\section{Consent for publication}

Not applicable.

\section{Competing interests}

The authors have no conflicts of interest to disclose.

\section{References}

1. WHO. Mental health and psychosocial considerations during the COVID-19 outbreak.

2. Boyraz G, Legros DN, Tigershtrom A. COVID-19 and traumatic stress: The role of perceived vulnerability, COVID-19-related worries, and social isolation. Journal of Anxiety Disorders 2020;76:102307.

3. Sawyer SM, Azzopardi PS, Wickremarathne D, et al. The age of adolescence. The Lancet Child \& Adolescent Health 2018;2:223-228. 
4. Fegert JM, Vitiello B, Plener PL, et al. Challenges and burden of the Coronavirus 2019 (COVID-19) pandemic for child and adolescent mental health: a narrative review to highlight clinical and research needs in the acute phase and the long return to normality. Child and Adolescent Psychiatry and Mental Health 2020;14:20.

5. Kessler RC, Berglund P, Demler O, et al. Lifetime Prevalence and Age-of-Onset Distributions of DSM-IV Disorders in the National Comorbidity Survey Replication. Archives of General Psychiatry 2005;62:593-602.

6. Steinberg L, Morris AS. Adolescent Development. Annual Review of Psychology 2001;52:83-110.

7. Charles NE, Strong SJ, Burns LC, et al. Increased mood disorder symptoms, perceived stress, and alcohol use among college students during the COVID-19 pandemic. Psychiatry research 2021;296:113706.

8. Choi J, Park Y, Kim H-E, et al. Daily Life Changes and Life Satisfaction among Korean School-Aged Children in the COVID-19 Pandemic. International Journal of Environmental Research and Public Health 2021;18:3324.

9. van der Laan SEl, Finkenauer C, Lenters VC, et al. Gender-Specific Changes in Life Satisfaction After the COVID-19-Related Lockdown in Dutch Adolescents: A Longitudinal Study. Journal of Adolescent Health 2021.

10. Wunsch K, Nigg C, Niessner C, et al. The Impact of COVID-19 on the Interrelation of Physical Activity, Screen Time and Health-Related Quality of Life in Children and Adolescents in Germany: Results of the Motorik-Modul Study. Children 2021;8:98.

11. Williams L, Rollins L, Young D, et al. What have we learned about positive changes experienced during COVID-19 lockdown? Evidence of the social patterning of change. PloS one 2021;16:e0244873.

12. Chen S, Bonanno GA. Psychological adjustment during the global outbreak of COVID-19: A resilience perspective. Psychological Trauma: Theory, Research, Practice, and Policy 2020;12:S51-S54.

13. Smith BW, Dalen J, Wiggins K, et al. The brief resilience scale: Assessing the ability to bounce back. Int J Behav Med 2008;15:194200.

14. Paredes MR, Apaolaza V, Fernandez-Robin C, et al. The impact of the COVID-19 pandemic on subjective mental well-being: The interplay of perceived threat, future anxiety and resilience. Personality and Individual Differences 2021;170:110455.

15. Leiner DJ. Too Fast, too Straight, too Weird: Non-Reactive Indicators for Meaningless Data in Internet Surveys. Survey Research Methods 2019;13:229-248.

16. Lu Z, Jiang Y, Shen C, et al. "Positive Energy": Perceptions and Attitudes Towards COVID-19 Information on Social Media in China. Proc ACM Hum-Comput Interact 2021;5:Article 177.

17. Cao J. Research on the Path to Teach Ideological and Political Theory Courses on Patriotism Education in Universities During the Fight Against COVID-19 Pandemic. 6th Annual International Conference on Social Science and Contemporary Humanity Development (SSCHD 2020); 2021; Atlantis Press; 2021. 62-67.

18. Goldberg D, Williams P. A user's guide to the General Health Questionnaire: Windsor, UK: NFER-Nelson, 1988.

19. Li J-B, Yang A, Dou K, et al. Self-Control Moderates the Association Between Perceived Severity of Coronavirus Disease 2019 (COVID19) and Mental Health Problems Among the Chinese Public. International Journal of Environmental Research and Public Health 2020;17:4820.

20. Li J-B, Yang A, Dou K, et al. Chinese public's knowledge, perceived severity, and perceived controllability of COVID-19 and their associations with emotional and behavioural reactions, social participation, and precautionary behaviour: a national survey. BMC Public Health 2020;20:1589.

21. Muthén LK, Muthén BO. Mplus user's guide (7th ed.): Los Angeles, CA: Muthén \& Muthén, 1998-2012.

22. Akaike H. A new look at the statistical model identification. IEEE Transactions on Automatic Control 1974;19:716-723.

23. Schwarz G. Estimating the Dimension of a Model. The Annals of Statistics 1978;6:461-464, 464.

24. Lo Y, Mendell NR, Rubin DB. Testing the number of components in a normal mixture. Biometrika 2001;88:767-778.

25. Arminger G, Stein P, Wittenberg J. Mixtures of conditional mean- and covariance-structure models. Psychometrika 1999;64:475-494.

26. Asparouhov T, Muthén B. Auxiliary Variables in Mixture Modeling: Three-Step Approaches Using Mplus. Structural Equation Modeling: A Multidisciplinary Journal 2014;21:329-341.

27. Nylund KL, Asparouhov T, Muthén BO. Deciding on the Number of Classes in Latent Class Analysis and Growth Mixture Modeling: A Monte Carlo Simulation Study. Structural Equation Modeling: A Multidisciplinary Journal 2007;14:535-569.

28. Hipp JR, Bauer DJ. Local solutions in the estimation of growth mixture models. Psychological Methods 2006;11:36-53.

29. Stanley L, Kellermanns FW, Zellweger TM. Latent Profile Analysis:Understanding Family Firm Profiles. Family Business Review 2017;30:84-102. 
30. Pyszczynski T, Lockett M, Greenberg J, et al. Terror Management Theory and the COVID-19 Pandemic. Journal of Humanistic Psychology 2021;61:173-189.

31. Dong H, Yang F, Lu X, et al. Internet Addiction and Related Psychological Factors Among Children and Adolescents in China During the Coronavirus Disease 2019 (COVID-19) Epidemic. Frontiers in Psychiatry 2020;11.

32. Masten AS. Ordinary magic: Resilience processes in development. American Psychologist 2001;56:227-238.

33. Kealy D, Rice SM, Seidler ZE, et al. Reflective functioning and men's mental health: Associations with resilience and personal growth initiative. Stress Health 2021.

34. Finstad GL, Giorgi G, Lulli LG, et al. Resilience, Coping Strategies and Posttraumatic Growth in the Workplace Following COVID-19: A Narrative Review on the Positive Aspects of Trauma. International Journal of Environmental Research and Public Health 2021;18:9453.

35. Park CL. Making sense of the meaning literature: An integrative review of meaning making and its effects on adjustment to stressful life events. Psychological Bulletin 2010;136:257-301.

36. Statistics NBo. Population and its composition.

\section{Tables}

Table 1 Summary of Latent Profile Models

\begin{tabular}{|c|c|c|c|c|c|c|c|c|c|}
\hline & $\begin{array}{l}\text { Log } \\
\text { Likelihood }\end{array}$ & $\begin{array}{l}\text { Number } \\
\text { of } \\
\text { Free } \\
\text { Parameter }\end{array}$ & AIC & $\mathrm{BIC}$ & $\mathrm{aBIC}$ & Entropy & $\begin{array}{l}\text { LMRT } \\
p \\
\text { value }\end{array}$ & $\begin{array}{l}\text { BLRT } \\
p \\
\text { value }\end{array}$ & $\begin{array}{l}\text { Class size } \\
\text { per profile }\end{array}$ \\
\hline $\begin{array}{l}1 \\
\text { Profile }\end{array}$ & -61123.07 & 38 & 122322.13 & 122544.45 & 122423.71 & - & - & - & 2567 \\
\hline $\begin{array}{l}2 \\
\text { Profiles }\end{array}$ & -53446.73 & 58 & 107009.47 & 107348.80 & 107164.51 & 0.92 & $\begin{array}{l}< \\
.001\end{array}$ & $\begin{array}{l}< \\
.001\end{array}$ & $1434 / 1133$ \\
\hline $\begin{array}{l}3 \\
\text { Profiles }\end{array}$ & -49960.22 & 78 & 100076.43 & 100532.77 & 100284.94 & 0.93 & $\begin{array}{l}< \\
.001\end{array}$ & $\begin{array}{l}< \\
.001\end{array}$ & $854 / 442 / 1271$ \\
\hline $\begin{array}{l}4 \\
\text { Profiles }\end{array}$ & -48553.91 & 98 & 97303.82 & 97877.16 & 97565.79 & 0.95 & .533 & $\begin{array}{l}< \\
.001\end{array}$ & $62 / 413 / 932 / 1160$ \\
\hline
\end{tabular}

Note: bolded entries represent the solution chosen in this study.

Table 2 Means, Standard Deviations, and MANOVA Tests for Indicators among the Three Profiles 


\begin{tabular}{|c|c|c|c|c|c|c|c|c|c|}
\hline \multirow[t]{2}{*}{ Items } & \multicolumn{2}{|c|}{ Profile 1} & \multicolumn{2}{|c|}{ Profile 2} & \multicolumn{2}{|c|}{ Profile 3} & \multirow{2}{*}{$\begin{array}{l}F(2, \\
2564)\end{array}$} & \multirow[t]{2}{*}{$p$} & \multirow[t]{2}{*}{$\eta_{p}^{2}$} \\
\hline & M & SD & M & SD & M & SD & & & \\
\hline 1. More quality time with family members & 3.03 & 0.78 & 4.51 & 0.73 & 3.53 & 0.78 & 533.63 & $\begin{array}{l}<.001 \\
.001\end{array}$ & .29 \\
\hline 2. Improved relationships with family & 2.90 & 0.66 & 4.44 & 0.77 & 3.39 & 0.71 & 699.64 & $\hat{.}_{.001}$ & .35 \\
\hline 3. Improved relationships with friends & 2.94 & 0.62 & 4.40 & 0.83 & 3.34 & 0.69 & 653.81 & $<.001$ & .34 \\
\hline $\begin{array}{l}\text { 4. Pay more attention to personal physical } \\
\text { health }\end{array}$ & 3.50 & 0.76 & 4.81 & 0.46 & 4.10 & 0.60 & 632.62 & $<.001$ & .33 \\
\hline $\begin{array}{l}\text { 5. Have more time to explore and cultivate } \\
\text { new hobbies }\end{array}$ & 3.03 & 0.73 & 4.62 & 0.64 & 3.63 & 0.73 & 725.88 & $<.001$ & .36 \\
\hline 6. Have better quality of sleep & 2.71 & 0.70 & 4.29 & 0.94 & 3.12 & 0.76 & 609.41 & $\begin{array}{l}<.001 \\
.001\end{array}$ & .32 \\
\hline 7. Become more gratitude & 3.14 & 0.71 & 4.78 & 0.44 & 3.92 & 0.61 & 1052.79 & $\begin{array}{l}<. \\
.001\end{array}$ & .45 \\
\hline $\begin{array}{l}\text { 8. Live a healthier life (e.g., healthier diet, } \\
\text { less smoking \& drinking) }\end{array}$ & 3.04 & 0.71 & 4.79 & 0.48 & 3.83 & 0.64 & 1122.81 & $<.001$ & .47 \\
\hline $\begin{array}{l}\text { 9. Spent less time on screens or devices } \\
\text { outsides of work/study hours }\end{array}$ & 2.46 & 0.79 & 4.13 & 1.09 & 2.94 & 0.87 & 518.70 & $<.001$ & .29 \\
\hline $\begin{array}{l}\text { 10. Be more appreciative of your current } \\
\text { work/study }\end{array}$ & 2.96 & 0.67 & 4.70 & 0.62 & 3.72 & 0.63 & 1094.71 & $\begin{array}{l}< \\
.001\end{array}$ & .46 \\
\hline $\begin{array}{l}\text { 11. Become more helpful (e.g., donated } \\
\text { goods, do voluntary work) }\end{array}$ & 3.21 & 0.64 & 4.81 & 0.45 & 3.95 & 0.55 & 1193.33 & $<.001$ & .48 \\
\hline 12. Become more respectful to others & 3.21 & 0.61 & 4.83 & 0.42 & 4.01 & 0.51 & 1413.58 & $<.001$ & .52 \\
\hline $\begin{array}{l}\text { 13. Become more concerned about your } \\
\text { country's and international situations }\end{array}$ & 3.70 & 0.73 & 4.91 & 0.33 & 4.29 & 0.54 & 657.61 & $\begin{array}{l}<.001 \\
.001\end{array}$ & .34 \\
\hline 14. Cherish the life even more & 3.64 & 0.76 & 4.94 & 0.29 & 4.40 & 0.52 & 803.40 & $\begin{array}{l}<.001 \\
.001\end{array}$ & .39 \\
\hline 15. Become more patriotic & 3.84 & 0.81 & 4.96 & 0.19 & 4.50 & 0.53 & 570.77 & $<.001$ & .31 \\
\hline 16. Found greater meaning in your own life & 3.08 & 0.64 & 4.89 & 0.34 & 4.01 & 0.61 & 1492.07 & $\begin{array}{l}<.001 \\
.001\end{array}$ & .54 \\
\hline $\begin{array}{l}\text { 17. Experience more positive emotions (e.g., } \\
\text { happy, joyful, excited) }\end{array}$ & 2.90 & 0.60 & 4.88 & 0.33 & 3.87 & 0.65 & 1710.68 & $<.001$ & .57 \\
\hline $\begin{array}{l}\text { 18. Become more optimistic about the } \\
\text { future }\end{array}$ & 2.94 & 0.60 & 4.90 & 0.31 & 3.98 & 0.60 & 1917.75 & $<.001$ & .60 \\
\hline 19. Feel stronger well-being & 2.97 & 0.61 & 4.89 & 0.32 & 3.95 & 0.63 & 1697.65 & $\begin{array}{l}<.001 \\
.001\end{array}$ & .57 \\
\hline
\end{tabular}

Table 3 Logistic Regression of Class Membership on Predictors 


\begin{tabular}{|c|c|c|c|c|c|}
\hline Predictors & Estimate & $S E$ & $p$ & OR & OR $95 \% \mathrm{Cl}$ \\
\hline \multicolumn{6}{|l|}{ Profile 1 (reference group) vs. Profile 2} \\
\hline Resilience & 0.70 & 0.13 & $<.001$ & 2.01 & {$[1.56,2.60]$} \\
\hline Sex $(1=$ male, 2 = female $)$ & -0.05 & 0.13 & .698 & 0.95 & {$[0.73,1.23]$} \\
\hline Age & -0.13 & 0.03 & $<.001$ & 0.88 & {$[0.83,0.93]$} \\
\hline Relation with COVID-19 ( 1 = related, 2 = non-related $)$ & 0.91 & 0.53 & .089 & 2.48 & {$[0.88,7.02]$} \\
\hline History of physical illness $(1=$ yes, $2=$ no $)$ & 0.51 & 0.34 & .132 & 1.67 & {$[0.86,3.24]$} \\
\hline History of psychiatric illness ( $1=$ yes, $2=$ no) & -0.41 & 0.68 & .552 & 0.66 & {$[0.18,2.52]$} \\
\hline Current physical health status & 0.84 & 0.10 & $<.001$ & 2.32 & {$[1.90,2.82]$} \\
\hline \multicolumn{6}{|l|}{ Profile 1 (reference group) vs. Profile 3} \\
\hline Resilience & 0.36 & 0.10 & $<.001$ & 1.43 & {$[1.18,1.74]$} \\
\hline Sex $(1=$ male, $2=$ female $)$ & 0.26 & 0.11 & .012 & 1.30 & {$[1.05,1.61]$} \\
\hline Age & 0.01 & 0.02 & .708 & 1.01 & {$[0.97,1.05]$} \\
\hline Relation with COVID-19 (1 = related, 2 = non-related $)$ & 0.82 & 0.36 & .020 & 2.27 & {$[1.12,4.60]$} \\
\hline History of physical illness $(1=$ yes, $2=$ no $)$ & 0.13 & 0.19 & .513 & 1.14 & {$[0.78,1.65]$} \\
\hline History of psychiatric illness ( $1=$ yes, $2=$ no) & 0.20 & 0.50 & .694 & 1.22 & {$[0.46,3.25]$} \\
\hline Current physical health status & 0.49 & 0.07 & $<.001$ & 1.63 & {$[1.42,1.87]$} \\
\hline \multicolumn{6}{|l|}{ Profile 3 (reference group) vs. Profile 2} \\
\hline Resilience & 0.34 & 0.11 & .003 & 1.40 & {$[1.13,1.74]$} \\
\hline Sex $(1=$ male, 2 = female $)$ & -0.32 & 0.12 & .011 & 0.73 & {$[0.57,0.92]$} \\
\hline Age & -0.13 & 0.03 & $<.001$ & 0.88 & {$[0.83,0.93]$} \\
\hline Relation with COVID-19 (1 = related, 2 = non-related $)$ & 0.09 & 0.55 & .873 & 1.09 & {$[0.37,3.22]$} \\
\hline History of physical illness $(1=$ yes, $2=$ no $)$ & 0.39 & 0.34 & .253 & 1.48 & {$[0.76,2.88]$} \\
\hline History of psychiatric illness ( $1=$ yes, $2=$ no) & -0.61 & 0.65 & .350 & 0.54 & {$[0.15,1.94]$} \\
\hline Current physical health status & 0.36 & 0.09 & $<.001$ & 1.43 & {$[1.20,1.71]$} \\
\hline
\end{tabular}

